\title{
An Energy Efficient Broadcasting for Mobile Devices Using a Cache Scheme*
}

\author{
Kook-Hee Han ${ }^{1}$, Jai-Hoon Kim ${ }^{1}$, Young-Bae $\mathrm{Ko}^{2}$, and Won-Sik Yoon ${ }^{2}$ \\ ${ }^{1}$ Graduate School of Information and Communication, \\ ${ }^{2}$ College of Information Technology, \\ Ajou University \\ \{justuniq, jaikim, youngko, wsyoon\}@ajou.ac.kr
}

\begin{abstract}
Broadcasting mechanisms have been widely used to transfer information to a large number of clients. Most of the broadcast schemes try to minimize the average "access time". In this paper, we present a broadcasting mechanism which uses a cache to reduce not only access time but also energy consumption. There is a trade-off between energy saving by accessing data in cache and energy consumption by receiving broadcast data to update cache. Therefore, we determine the optimal size of cache to minimize energy consumption according to information access patterns and update characteristics.
\end{abstract}

\section{Introduction}

Transferring the information of common interest to mobile users is an important issue in personal wireless communications such as stock trading systems, weather information systems, and parking information systems. In these systems, broadcast mechanisms can be efficiently used, in which a broadcast server (e.g., satellite and base station) transfers the information of common interest to a large number of mobile users. An asymmetric environment is common in such systems. The downstream communication capacity (from server to clients) is much greater than the upstream capacity (from clients to server) [1]. Many schemes [1,2,3] have been proposed to broadcast information efficiently to a large number of users, and their main purpose is to minimize the average "access time" for the information needed. The access time is the time amount required for a client to wait for information that the client needs.

Mobile devices being used in personal wireless communications such as PDA, Palmtops, etc. are powered by small batteries without directly connecting to fixed power sources. Many hardware and software schemes are proposed and implemented to overcome such a power constraint. As one of the solutions, the index-based organization of data transmitted over wireless channels is proposed to reduce power

\footnotetext{
This work was supported by grant no. R05-2003-000-10607-0 and R01-2003-00-0794-0 from Korea Science and Engineering Foundation, by ITA Professorship for Visiting Faculty Positions in Korea (International Joint Research Project) from Ministry of Information and Communication in Korea, by Korea Research Foundation Grant (KRF-2003-003-D00375), and by University IT Research Center Project.
} 
consumption. Clients are interested in obtaining the individual data items from the broadcast[4,5]. If a directory index has information when a specific data item is transferred in the broadcast, then each client needs listening the channel in active mode selectively to obtain the required data, while in doze mode during the rest time to reduce energy consumption.

In this paper, we present a new index-based broadcasting mechanism using cache to reduce energy consumption. There is a trade-off between energy saving by accessing data in cache and energy consumption caused by cache memory itself and frequent cache update through broadcast. We propose an algorithm to decide the optimal size of cache to minimize energy consumption of mobile device in broadcast networks.

\section{Index-Based Broadcast Using Cache Scheme}

In general, a mobile node consumes much of its energy during data communication (e.g., broadcast). Table 1 shows the difference of energy dissipation between data access via wireless link and data access from cache memory [6,7]. In cache mechanisms, a mobile node holds data units which are most likely to be used in the future. It can certainly reduce energy consumption as well as access time.

Table 1. Per bit of energy cost

\begin{tabular}{c|c} 
Operation & Energy Dissipate \\
\hline Broadcast & $50 \mathrm{~nJ} / \mathrm{bit}$ \\
Caching & $1.65 \mathrm{~nJ} / \mathrm{bit}$
\end{tabular}

When data in server is changed, data broadcast is needed to update cache data. In some situations, data update occurs very frequently, and it can cause increased energy consumption in mobile node to receive update data. Thus, there is a trade-off between energy saving by using cache instead of receiving data via wireless link and increased energy consumption caused by frequent broadcast for data update. We need to determine the optimal size of cache that minimizes energy consumption. We assume that popularity of data usage (locality of data access) follows zipfian distribution $[8,9]$.

Equation (1) belong represents an expected power consumption $\left(P_{\text {broadcass }}\right)$ to receive broadcast data not in the cache of mobile device. $K_{l}$ represents energy consumption to access one data unit not in the cache using broadcast, where $c$ is the cache size.

In Equation (2), $K_{2}$ represents energy consumption to access one unit of data in cache. Remind that, as shown in Table 1, when the mobile node holds needed data in the cache, it consumes much energy. Of course, cache update requires additional energy consumption as shown in Equation (3). In Equations (2) and (3), $T$ denotes an average update interval of cache. Now in Equation (4), $P_{\text {total }}$ represents overall expected energy consumption when mobile device uses $c$ units of cache. We can measure total energy consumption $P_{\text {total }}$ as the sum of $P_{\text {broadcs' }}, P_{\text {cache }}$ and $P_{\text {update }}$. 


$$
\begin{gathered}
P_{\text {broadcast }}=K_{1} \times \sum_{x=c+1}^{n} \frac{1}{x^{\theta}} \\
P_{\text {cache }}=K_{2} \times \sum_{x=1}^{c} \frac{1}{x^{\theta}} \\
P_{\text {update }}=\frac{K_{1}}{T} \times c \\
P_{\text {total }}=P_{\text {broadcast }}+P_{\text {cache }}+P_{\text {update }}=K_{1}\left(\sum_{x=c+1}^{n} \frac{1}{x^{\theta}}+\frac{c}{T}\right)+K_{2} \sum_{x=1}^{c} \frac{1}{x^{\theta}}
\end{gathered}
$$

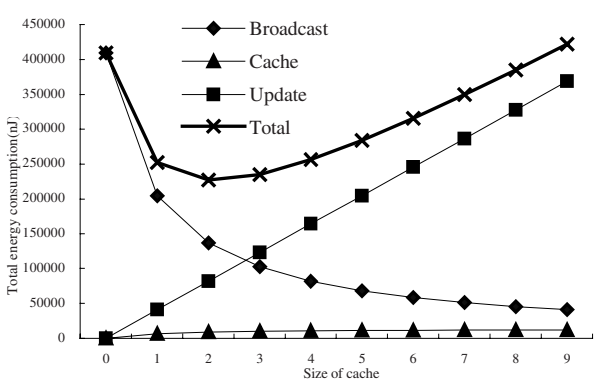

Fig. 1. Energy consumption in mobile node (when, $\mathrm{T}=10, \theta=2$ )

Consequently, we find the optimal size of cache that minimizes total energy consumption for the broadcast scheme with cache.

Fig. 1 shows the overall energy consumption from broadcast, cache, and cache update, respectively, to access total 1024 bytes per period T. As shown in Fig. 1, mobile node consumes the least energy when 2 units of cache are used by mobile device in the system parameters $(T=10, \theta=2)$.

The result can be changed by differentiating update rate and the distribution of data access popularity $\theta$. Therefore, it is needed to reflect data update rate and data popularity to select the optimal size of cache. As shown in Fig. 2 and 3, the amount of energy consumption increases as the update rate $(1 / T)$ and data popularity $(\theta)$ decreases.

In Fig. 2, we observe energy consumption as the size of cache increases for different cache update rates. Cache update rate $(1 / T)$ represents how often cache update occurs on average. As shown in Fig. 2, energy consumption increases as the cache is frequently updated. Since each cache update needs additional energy consumption to receive broadcast data.

Fig. 3 shows energy consumption for different popularity $(\theta)$ of data access. As a result, energy consumption decreases as the data request popularity increases by accessing most of data in the cache. 


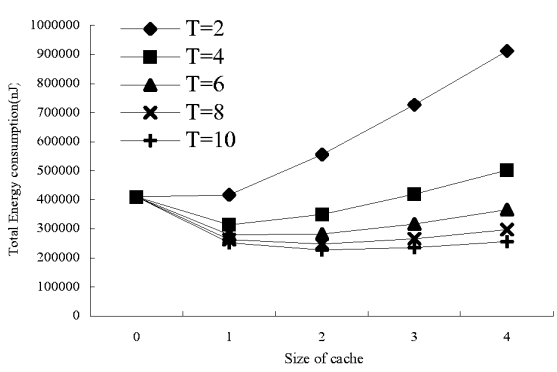

Fig. 2. Energy consumption in mobile node (when $\theta=2$ )

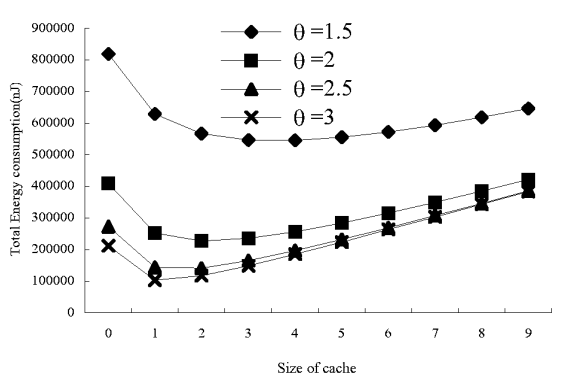

Fig. 3. Comparison of energy consumption (when $T=10$ )

\section{Conclusion}

This paper presents energy saving broadcast using cache. We found that the optimal size of cache exists to minimize energy consumption. Concentration on specific data access, and data updating rate are important factor to decide the size of cache. We can decide the size of cache to minimize energy consumption for each system parameters.

\section{References}

1. S. Hameed and N. H. Vaidya, "Efficient Algorithms for Scheduling Data Broadcast," ACM/Baltzer Wireless Networks (WINET), May 1999.

2. T. Imielinski, S. Viswanathan, and B. R. Badrinath, "Data on Air: Organization and Access," IEEE Transactions on Knowledge and Data Engineering, Vol. 9, No. 3, May/June 1997.

3. S. Acharya, M. Franklin, and S. Zdonik, "Dissemination-based data delivery using broadcast disks," IEEE Personal Communication, pp. 50-60, December 1995.

4. J. Dukes-Schlossberg, Y. Lee, N. Lehrer, "IIDS: Intellegent Information Dissemination Server," Proc. of IEEE MILCOM '97, Vol. 2, pp. 635-9.

5. R. Jain and J. Werth, "Airdisks and airRAID : Modeling and scheduling periodic wireless data broadcast (extended abstract)," Tech. Rep. DIMACS Tech. Report 95-11, Rutgers University, May 1995.

6. W. R. Heinzelman, A. Chandrakasan, H. Balakrishnan, "Energy-efficient communication protocol for wireless microsensor networks," Hawaii International Conference on Systems Sciences, 2000.

7. Mobile DRAM memory specification, http://www.sec.co.kr

8. John L. Casti, "Five More Golden Rules: Knots, Codes, Chaos, and Other Great Theories of 20th-Century Mathematics," John Wiley \& Sons Inc, 2000.

9. Zipf Curves and Website Popularity, http: //www. useit. com 